ARTICLE

\title{
Parkinson's disease associated mutation E46K of $\alpha$-synuclein triggers the formation of a distinct fibril structure
}

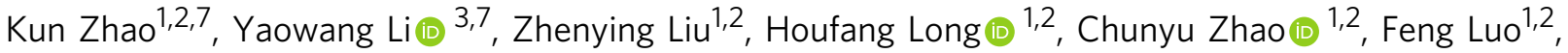 \\ Yunpeng Sun ${ }^{1,2}$, Youqi $\mathrm{TaO}^{4}$, Xiao-dong Su${ }^{5}$, Dan Li (i) ${ }^{4,6 凶}$, Xueming Li(i) ${ }^{3 凶} \&$ Cong Liu (i) ${ }^{1,2 凶}$
}

Amyloid aggregation of $\alpha$-synuclein ( $\alpha$-syn) is closely associated with Parkinson's disease (PD) and other synucleinopathies. Several single amino-acid mutations (e.g. E46K) of $\alpha$-syn have been identified causative to the early onset of familial PD. Here, we report the cryo-EM structure of an $\alpha$-syn fibril formed by $N$-terminally acetylated E46K mutant $\alpha$-syn (Ac-E46K). The fibril structure represents a distinct fold of $\alpha$-syn, which demonstrates that the E46K mutation breaks the electrostatic interactions in the wild type (WT) $\alpha$-syn fibril and thus triggers the rearrangement of the overall structure. Furthermore, we show that the Ac-E46K fibril is less resistant to harsh conditions and protease cleavage, and more prone to be fragmented with an enhanced seeding capability than that of the WT fibril. Our work provides a structural view to the severe pathology of the PD familial mutation E46K of $\alpha$-syn and highlights the importance of electrostatic interactions in defining the fibril polymorphs.

\footnotetext{
${ }^{1}$ Interdisciplinary Research Center on Biology and Chemistry, Shanghai Institute of Organic Chemistry, Chinese Academy of Sciences, Shanghai 201210, China. ${ }^{2}$ University of Chinese Academy of Sciences, Beijing 100049, China. ${ }^{3}$ Key Laboratory of Protein Sciences (Tsinghua University), Ministry of Education, School of Life Sciences, Tsinghua University, Beijing 100084, China. ${ }^{4}$ Bio-X Institutes, Key Laboratory for the Genetics of Developmental and Neuropsychiatric Disorders, Ministry of Education, Shanghai Jiao Tong University, Shanghai 200030, China. ${ }^{5}$ State Key Laboratory of Protein and Plant Gene Research, and Biomedical Pioneering Innovation Center (BIOPIC), School of Life Sciences, Peking University, Beijing 100871, China. ${ }^{6}$ Bio-X-Renji Hospital Research Center, Renji Hospital, School of Medicine, Shanghai Jiao Tong University, Shanghai 200240, China. ${ }^{7}$ These authors contributed equally: Kun Zhao,

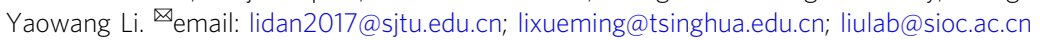


D eposition of a-syn amyloid fibrils in Lewy bodies (LB) and Lewy neuritis (LN) is a common histological hallmark of Parkinson's disease (PD) and synucleinopathies such as dementia with Lewy bodies (DLB) and multiple system atrophy $(\mathrm{MSA})^{1-3}$. Accumulating evidence shows that $\alpha$-syn amyloid fibrils serve as prion-like seeds for the propagation and cell-to-cell transmission of the pathological entity of $\alpha-\operatorname{syn}^{4,5}$. The spread of pathological $a$-syn fibril is closely associated with the disease progression $^{6,7}$. Several single point mutations and genomic duplication or triplication of SNCA, the gene encoding a-syn, have been identified to be causative to the familial forms of the diseases with a broad spectrum of distinctive clinical symptoms ${ }^{8-12}$. Moreover, the hereditary mutations exhibit exacerbated pathology in various cellular and PD-like animal models ${ }^{13,14}$. Intriguingly, the cryo-EM structure of full-length wild-type (WT) a-syn fibril demonstrates that in the five mutation sites found in familial PD, four of them, i.e. E46K, A53T/E, G51D, and H50Q, are located at the protofilamental interface of the WT fibril ${ }^{15,16}$, which indicates that these mutations may alter the fibril structure and consequently influence $\alpha$-syn amyloid aggregation and PD pathology.

a-Syn hereditary mutation E46K was originally identified from a Spanish family with autosomal dominant parkinsonism ${ }^{11}$. The clinical phenotype of E46K patient features rapid and severe disease progression with early onset of the parkinsonism of $\mathrm{DLB}^{11}$. Previous studies have shown that the E46K mutation can enhance $\alpha$-syn fibril formation and $\alpha$-syn pathology in vitro and in cultured cells ${ }^{13,17,18}$. Solid-state NMR study has shown that the E46K mutation causes large conformational changes of the fibril structure $^{19}$.

In this work, we determine the cryo-EM structure of the $\mathrm{N}$ terminally acetylated E46K $\alpha$-syn (Ac-E46K) fibril at an overall resolution of $3.37 \AA$. The structure reveals a distinct fold of $\alpha$-syn, in which the electrostatic interactions in the WT fibril are broken and reconfigured due to the E46K mutation. We further show that the Ac-E46K fibril is less stable than the WT fibril under harsh conditions and protease cleavage, while the mutant fibril is more efficient in seeding amyloid fibril formation. This work provides a structural mechanism for the influence of E46K on the amyloid fibril formation of $a$-syn, and suggests that electrostatic interactions may serve as one of the driving force for the polymorphic fibril formation of a-syn.

\section{Results}

E46K mutation alters the morphology and stability of $\alpha$-syn fibril. To investigate the influence of E46K on a-syn fibril formation, we prepared recombinant full-length $\alpha$-syn with E46K mutation. Since in vivo $\alpha$-syn is generally acetylated at the $N$ terminus ${ }^{20,21}$, we modified the recombinant $\alpha$-syn with $\mathrm{N}$-terminal acetylation (Supplementary Fig. 1). The recombinant $\mathrm{N}$-acetylated E46K a-syn (termed as Ac-E46K) formed amyloid fibrils after incubation in the buffer containing $50 \mathrm{mM}$ Tris, $150 \mathrm{mM} \mathrm{KCl,} \mathrm{pH}$ 7.5 , at $37^{\circ} \mathrm{C}$ with agitation for 7 days. We first used the atomic force microscopy (AFM) to characterize the fibril morphology. The result showed that the Ac-E46K fibril features a different fibril structure from the N-acetylated WT (Ac-WT) fibril formed under the same condition $^{15}$ (Supplementary Fig. 2). The Ac-E46K fibril is approximately two times more twisted than the Ac-WT fibril with a half pitch of $\sim 64 \mathrm{~nm}$ in comparison with $\sim 120 \mathrm{~nm}$ of the Ac-WT. Moreover, the Ac-E46K fibril features a right-handed helical twist (Supplementary Fig. 2), which is distinct from the left-handed twist commonly found in the WT and other PD-familial mutant $\alpha$-syn fibrils ${ }^{15,16,22-24}$. The different fibril morphologies suggest different properties of the Ac-E46K and Ac-WT fibrils, which may associate with their different pathologies.
To identify the different properties of the Ac-E46K and Ac-WT fibrils, we first sought to test the stability of the fibrils by cold denaturation $^{25}$. The fibrils were cooled down and incubated at $0{ }^{\circ} \mathrm{C}$ for $48 \mathrm{~h}$. The result of circular dichroism (CD) spectroscopy showed that the Ac-E46K fibril lost its $\beta$-sheet structure significantly faster than that of the Ac-WT fibril (Fig. 1a). Consistently, negative-staining transmission microscopic (TEM) imaging showed a faster dissembling of the Ac-E46K fibril than that of the Ac-WT fibril (Supplementary Fig. 3a). Intriguingly, we noticed that the Ac-E46K fibril stored at $-80{ }^{\circ} \mathrm{C}$ underwent apparent fragmentation after thawing; in contrast, the Ac-WT fibril were generally stable, and fragmentation was only observed after several cycles of freeze-thaw (Supplementary Fig. 3b). CD spectra further showed that in eight cycles of freeze-thaw, the structure of Ac-WT fibril was well maintained with a consistent content of $\beta$-sheet structure; in contrast, the Ac-E46K fibril gradually lost its $\beta$-sheet structure as the freeze-thaw cycles increased (Fig. 1b). In consistence with the freeze-thaw experiment, as we sonicated the preformed fibrils (PFFs), we noticed that under the same sonication condition, the Ac-E46K fibril broke into significantly shorter fragments than that of the Ac-WT fibril visualized by TEM and AFM (Fig. 1c, d, and Supplementary Fig. 4). It has been shown that fibril fragmentation is a key factor in nucleation-depended fibrillation ${ }^{26}$. Thus, these results indicate that the Ac-E46K fibril is less stable than the Ac-WT fibril, and may gain an enhanced pathology.

Since $a$-syn fibrils propagate by seeding the amyloid fibril formation in the transfected neurons, we attempted to compare the seeding property of the Ac-E46K and Ac-WT PFFs. We sonicated the PFFs and confirmed a comparable amount of AcE46K and Ac-WT PFF seeds by SDS-PAGE (Supplementary Fig. 5a). Of note, although in the absence of PFF seeds, the fibrillation of both Ac-WT and Ac-E46K were weak in our assay (Supplementary Fig. 5b and c), we observed a faster aggregation of Ac-WT than that of the Ac-E46K (Supplementary Fig. 5d). On the contrary, in the presence of PFF seeds, Ac-E46K aggregated faster than Ac-WT (Fig. 1e). Consistently, the results of TEM and SDS-PAGE showed more amyloid fibrils formed in the seeded Ac-E46K samples than that of the Ac-WT (Supplementary Fig. 5b and c). These results indicate that the Ac-E46K PFF seeds are more potent than that of the Ac-WT in seeding a-syn fibril formation.

Furthermore, we compared the stability of the fibrils under proteinase $\mathrm{K}(\mathrm{PK})$ digestion. The result showed that the Ac-E46K fibril is digested significantly faster than that of the Ac-WT fibril (Fig. 1f). Together, these data indicate that the Ac-E46K fibril is less stable than the Ac-WT fibril in both physical and chemical treatments and more efficient in fragmentation and propagation.

Structure determination of the Ac-E46K a-syn fibril by cryoEM. To understand the structural basis underlying the different properties between the Ac-E46K and Ac-WT fibrils, we sought to determine the atomic structure of Ac-E46K fibril by cryo-EM. The Ac-E46K fibril sample was fixed on the carbon grid and frozen in liquid ethane. The cryo-EM micrographs were acquired at $\times 105,000$ magnification on the $300 \mathrm{keV}$ Titan Krios microscope equipped with the K2 Summit camera. 13,064 fibrils picked from 754 micrographs were used for the reconstruction of the Ac-E46K fibril (Table 1). The Ac-E46K fibril sample is morphologically homogeneous with one dominant species in the $2 \mathrm{D}$ classification of the fibrils (Supplementary Fig. 6). After helical reconstruction of the dominant fibril species by Relion, we obtained a 3D density map of the Ac-E46K fibril to an overall resolution of $3.37 \AA$ (Supplementary Fig. 7). The density map showed a right-handed helix with a width of $\sim 10 \mathrm{~nm}$ and a half helical pitch of $\sim 68 \mathrm{~nm}$ 
a
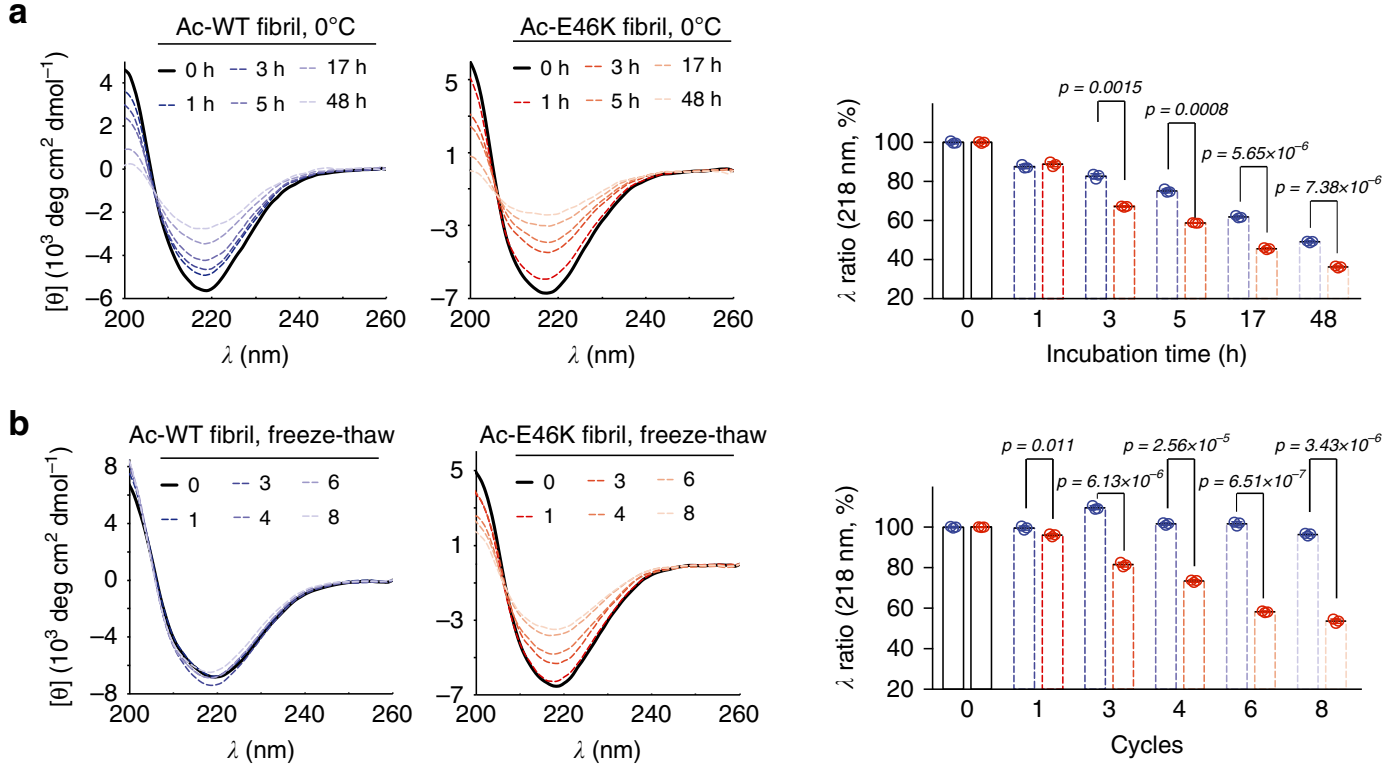
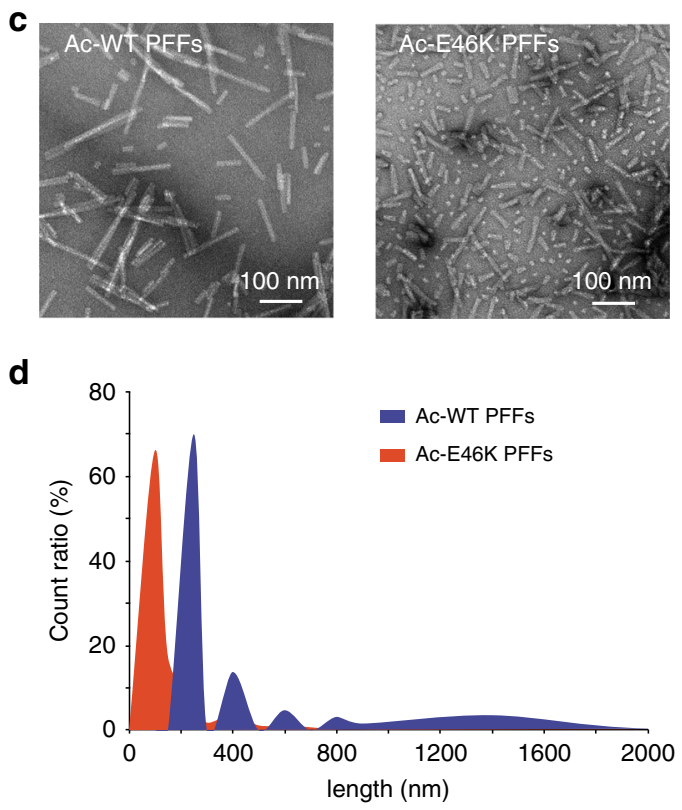

f

PK digestion $\left(30 \mathrm{~min}\right.$ at $\left.37^{\circ} \mathrm{C}\right)$

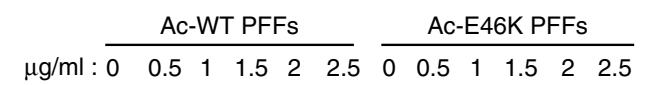

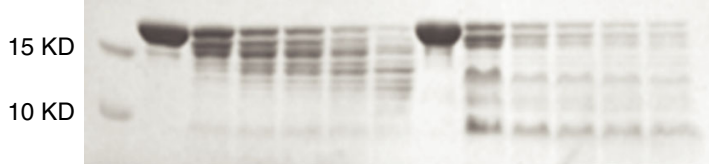

(Fig. 2), which is consistent with the AFM measurement (Supplementary Fig. 2). The fibril contains two protofilaments that intertwine along an approximate 2 -fold screw axis (Fig. 2). The helical twist between $\alpha$-syn subunits is $-179.37^{\circ}$ and the helical rise is $2.38 \AA$ (Fig. 2b, c, Table 1).

The Ac-E46K fibril exhibits a distinct fold of $\alpha$-syn fibril. The high quality cryo-EM density map allowed us to unambiguously
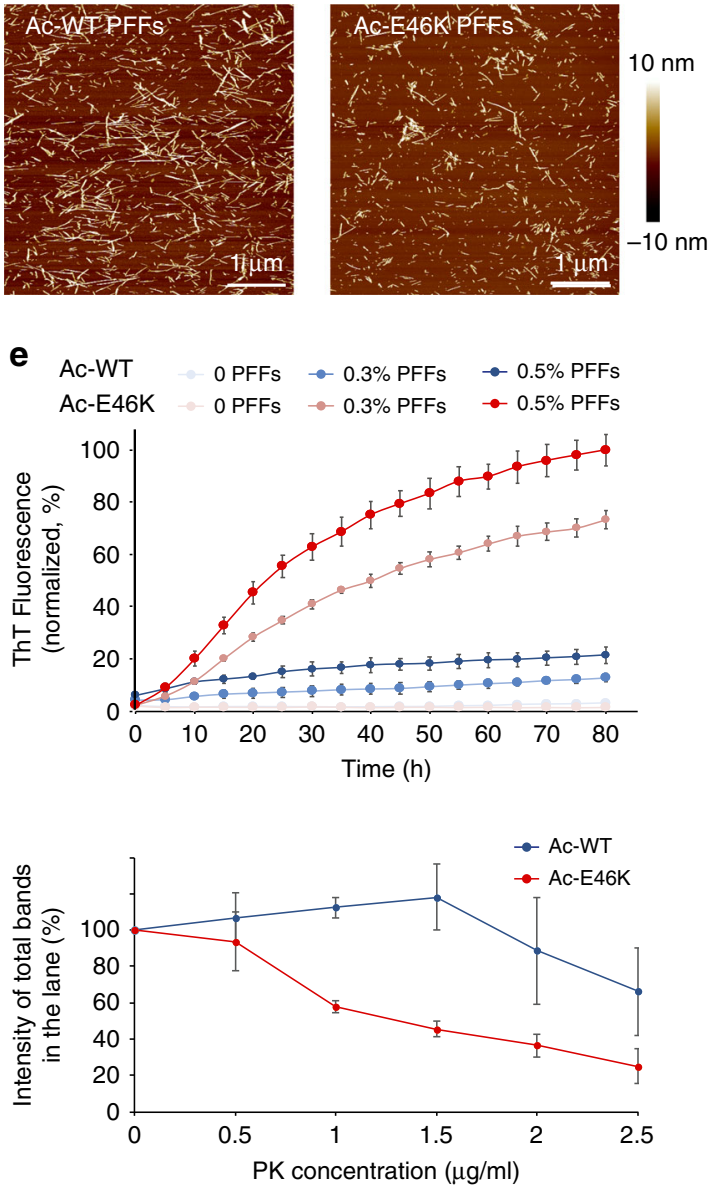

build an atomic structure model for the Ac-E46K fibril (Fig. 3a). This structure represents the fibril core (FC) of the Ac-E46K fibril consisting of residues 45-99 out of a total of 140 amino acids of a-syn (Fig. 3a), which is slightly smaller than the Ac-WT FC consisting of residues 37-99 formed under the same condition ${ }^{15}$. While, similar to that of the WT fibril, the N- and C-termini of Ac-E46K $\alpha$-syn remain flexible and are not visible by cryo-EM.

The Ac-E46K FC exhibits a serpentine fold, in which the topology of the $\beta$ strands is different from any reported $\alpha$-syn 
Fig. 1 Comparison of the stability of the Ac-WT and Ac-E46K $\boldsymbol{\alpha}$-syn fibrils. a Cold denaturation of $20 \mu \mathrm{M} \mathrm{Ac-WT}$ (blue) and Ac-E46K (red) $\alpha$-syn fibrils. $\mathrm{CD}$ spectra monitored at $0^{\circ} \mathrm{C}$ at different incubation time are shown on the left. $\mathrm{CD}$ signals at $218 \mathrm{~nm}$ are analyzed on the right. $\mathbf{b}$ Freeze-thaw denaturation of Ac-WT (blue) and Ac-E46K (red) fibrils. CD spectra obtained at different freeze-thaw cycles are shown on the left. CD signals at $218 \mathrm{~nm}$ are analyzed on the right. Data are shown as mean \pm s.d., $n=3$ biologically independent samples for both panels (a) and (b). Exact $p$ values of the unpaired, two-tailed Student's $t$ test are shown. c Negative-staining TEM (left) and AFM (right) images of $5 \mu$ M Ac-WT and Ac-E46K $\alpha$-syn PFFs after sonication. d Size distribution of sonicated Ac-WT and Ac-E46K PFFs. The lengths of the sonicated PFFs are measured by AFM. 300 fibrils were measured for each fibril sample. e ThT kinetic assay of the Ac-WT and Ac-E46K fibril formation with or without seeding. Mole percent of added PFF seeds are indicated. Data shown are mean \pm s.d., $n=3$. $\mathbf{f}$ PK digestion of the Ac-WT and Ac-E46K fibrils. The fibrils were incubated with indicated concentrations of PK at $37^{\circ} \mathrm{C}$ for $30 \mathrm{~min}$ (left). Intensities of the total protein bands of each lane on SDS-PAGE are analyzed on the right. Data shown are mean \pm s.d., $n=3$ biologically independent samples. Source data are provided as a Source Data file.

$\begin{array}{|ll|}\text { Table } 1 \text { Statistics of cryo-EM data collection and refinement. } \\ & \\ \text { NAME } & \text { Ac-E46K } \alpha \text {-syn fibril } \\ \text { PDB ID } & 6 \text { L4S } \\ \text { EMDB ID } & \text { EMD-0833 } \\ \text { DATA COLLECTION } & \\ \text { Magnification } & 105,000 \\ \text { Pixel size }(\AA) & 0.665 \\ \text { Defocus Range ( } \mu \text { m) } & -1.5 \text { to }-2.4 \\ \text { Voltage (kV) } & 300 \\ \text { Camera } & \text { K2 summit } \\ \text { Microscope } & \text { Titan Krios } \\ \text { Exposure time (s per frame) } & 0.25 \\ \text { Number of frames } & 32 \\ \left.\text { Total dose (e- per } \AA^{2}\right) & 50 \\ \text { RECONSTRUCTION } & \\ \text { Micrographs } & 754 \\ \text { Manually picked fibrils } & 13,064 \\ \text { Box size (pixel) } & 160 \\ \text { Inter-box distance }(\AA) & 19 \\ \text { Segments extracted } & 806,640 \\ \text { Segments after Class2D } & 49,902 \\ \text { Segments after Class3D } & 18,009 \\ \text { Resolution }(\AA) & 3.37 \\ \text { Map sharpening B-factor }\left(\AA{ }^{2}\right) & -97.805 \\ \text { Helical rise }(\AA) & 2.38 \\ \text { Helical twist }\left({ }^{\circ}\right) & -179.37 \\ \text { ATOMIC MODEL } & \\ \text { Initial model used (PDB ID) } & 2 \mathrm{NOA} \\ \text { Map sharpening B-factor }(\AA) & -90.924 \\ \text { Non-hydrogen atoms } & 2,268 \\ \text { Protein residues } & 330 \\ \text { Ligands } & 0 \\ \text { r.m.s.d. bond lengths } & 0.014 \\ \text { r.m.s.d. bond angles } & 1.359 \\ \text { All-atom clashscore } & 7.71 \\ \text { Rotamer outliers } & 0.00 \% \\ \text { Ramachandran Butliers } & 0.00 \% \\ \text { Ramachandran Allowed } & 5.66 \% \\ \text { Ramachandran Favored } & 94.34 \% \\ & \\ & \\ & \end{array}$

fibril structure ${ }^{15,16,22-24,27,28}$ (Fig. 3b). Compared to the structure of Ac-WT a-syn fibril, the major difference lies in the N-terminal region of the FC (termed as FC-N), which covers residues 37-59 in the Ac-WT fibril or 45-59 in the Ac-E46K fibril. In the Ac-WT structure, the FC-N region stretches and packs around the rest of the a-syn molecule (termed as FC-C) via interactions with T75, A76, A78, and K80 of FC-C (Fig. 3c). In contrast, in the Ac-E46K structure, this segment forms a $\beta$ hairpin and extends from the FC-C with interactions to the side chains of Q62, T64, and V66 (Fig. 3c).

Aside from the large conformational change of the FC-N, the FC-C regions of the Ac-E46K and Ac-WT fibrils adopt a similar

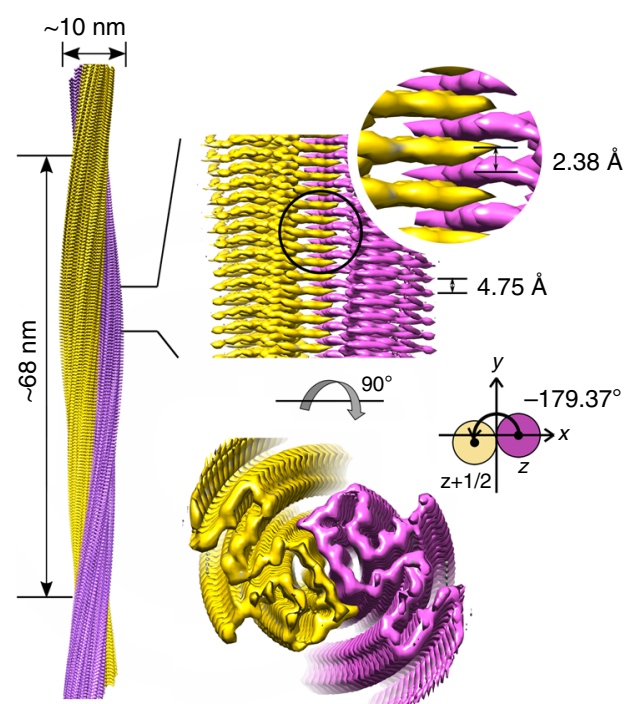

Fig. 2 Cryo-EM 3D reconstruction density map of the Ac-E46K $\alpha$-syn fibril. Fibril width, length of half pitch $\left(180^{\circ}\right.$ helical turn), helical rise and twist angle are indicated. The twist angle is graphically illustrated. The two intertwining protofilaments are colored in yellow and purple, respectively. Graphing was performed with UCSF Chimera v1.13.

topology with a Greek key-like fold (Fig. 3c); however, the two FC-C structures are very different with an r.m.s.d. (Ca atoms) of $5.558 \AA$ (Supplementary Fig. 8). In the Ac-WT fibril, a-syn subunits fold in a flat layer; in contrast, in the Ac-E46K fibril, $\beta 6$ and $\beta 7$ of the FC-C swap to the next layer to form inter-molecular side-chain interactions within the same protofilament (Fig. 3d). Thus, the single mutation of E46K entirely changes the a-syn fibril structure.

E46K rearranges the electrostatic interactions in the a-syn fibril. Since the overall structural rearrangement of $\alpha$-syn fibril is initiated by one single mutation of $\mathrm{E} 46 \mathrm{~K}$, we looked close to residue 46 in the Ac-WT and Ac-E46K fibrils. In the Ac-WT fibril, E46 forms a salt bridge with K80, which together with the salt bridge formed by E61 and K58, locks the FC-N with FC-C (Fig. 4a, b). In contrast, in the Ac-E46K fibril, the E46K mutation breaks the salt bridge of E46-K80, which results in the breaking of the other two essential electrostatic interactions (K58-E61 and K45-H50-E57) (Fig. 4a, b). Alternatively, in the Ac-E46K fibril, K80 forms inter-molecular salt bridge with E61 of the opposing a-syn subunit to involve in the fibril interface, and K45 directly forms salt bridge with E57 to stabilize the $\beta$-hairpin conformation of the FC-N (Fig. 4a, b).

The E46K mutation also leads to the disruption of the WT protofilament interface (Fig. 4c, d). Upon the flipping away of the FC-N from the fibril interface, a distinct fibril interface is formed. The exposed segments 74-79 of opposing a-syn subunits form 


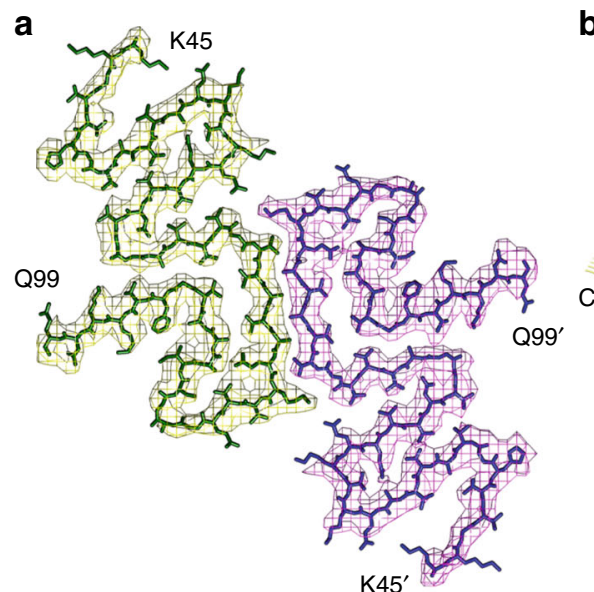

C

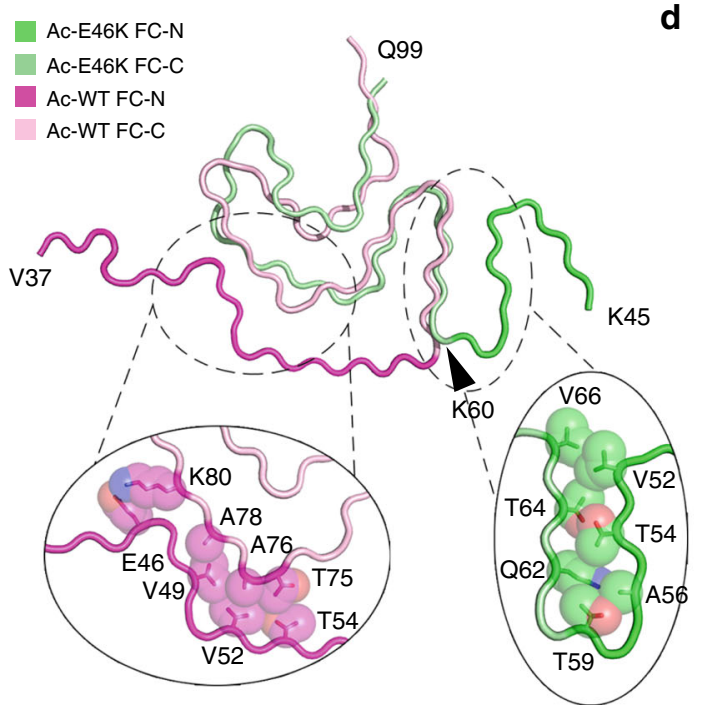

b
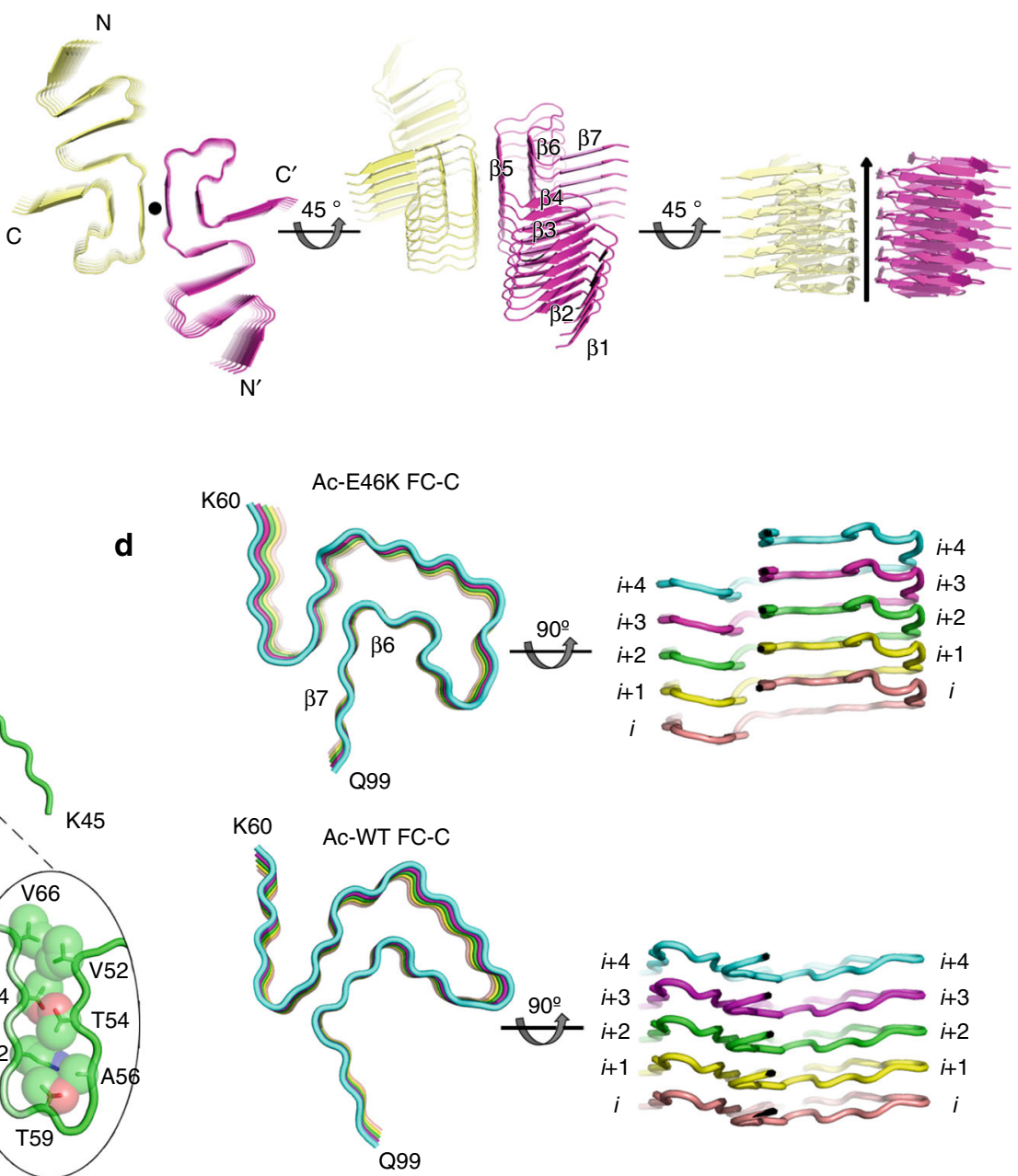

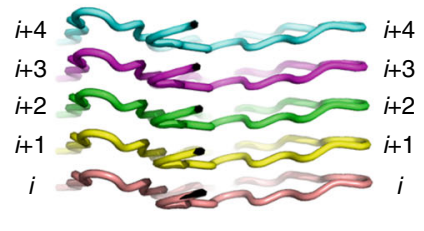

Fig. 3 Cryo-EM structure of the Ac-E46K $\boldsymbol{\alpha}$-syn fibril. a Top view of the Ac-E46K fibril. One layer of the structure is shown, which consists of two $\alpha$-syn molecules covering residues 45-99. The two molecules are colored differently. b Views of six layers of the Ac-E46K fibril are shown in cartoon. The two protofilaments are colored differently. The fibril axis is indicated. The $\beta$ strands are numbered and labeled. c Overlay of the $\alpha$-syn subunit structures of the Ac-E46K and Ac-WT fibrils. The two structures diverge mainly at the FC-N region. The interfaces between the FC-N and FC-C regions are shown in the zoom-in views. The residues involved in the interfaces are labeled and their side chains are shown in spheres. $\mathbf{d}$ Comparison of the FC-C region of the AcE46K and Ac-WT fibrils. Five layers of a single protofilament is shown. The structures are colored by different layers. The FC-C region adopts similar topology in the two structures, while their structural arrangements in the two fibrils are markedly different. The FC-C of the Ac-WT fibril folds in a flat layer. In contrast, in the Ac-E46K fibril, $\beta 6$ and $\beta 7$ swap to the next $\alpha$-syn molecule in the neighboring layer.

typical class I parallel in-register homo-steric zippers to mediate the inter-protofilamental interactions (Fig. 4d). The steric-zipper interface is stabilized by the hydrophobic interactions between the side chains of V74, A76, and A78 and flanked by the intermolecular salt bridges formed by K80-E61', which further lock the two protofilaments (Fig. 4a).

The release of FC-N from the fibril interface in the Ac-E46K fibril increases the flexibility of this segment, which exhibits higher $\mathrm{B}$-factors and poor density for tracing the $\mathrm{N}$-terminal residues 37-44 that are visible in the Ac-WT FC (Fig. 4c). Thus, the relatively loose packing of the Ac-E46K fibril in comparison with that of the Ac-WT fibril may explain the decreased stability and increased sensitivity of the Ac-E46K fibril to the environment, which may associate with the pathology of this PD familial mutation.

\section{Discussion}

Structural polymorphism is a common characteristic of amyloid fibrils formed by different amyloid proteins such as $\alpha$-syn, tau and $A \beta^{29-31}$. Different fibril polymorphs may represent different pathological entities that are associated with different subsets of neurodegenerative diseases ${ }^{32-34}$. Determination of polymorphic amyloid fibril structures is useful for the mechanistic understanding of amyloid pathology. In this work, we report the cryoEM structure of an $\alpha$-syn fibril with $\mathrm{N}$-terminal acetylation and familial mutation E46K found in parkinsonisms. The Ac-E46K asyn fibril exhibits a distinct structure that is distinct from the reported $\alpha$-syn polymorphs. Compared to the Ac-WT fibril prepared under the same condition, the E46K mutation breaks an important electrostatic interaction of E46-K80 and thus induces the reconfiguration of the overall structure. In the mutant fibril, the FC-N region that used to form the protofilamental interface in the Ac-WT fibril, flips away from the fibril interface and becomes less stable. In line with the structural information, we find that the Ac-E46K fibril is less stable upon both physical and chemical treatments. Furthermore, seeding experiments indicate that the instability and fragmentation-prone property of Ac-E46K fibril may lead to an increased capability of propagation. Of note, 


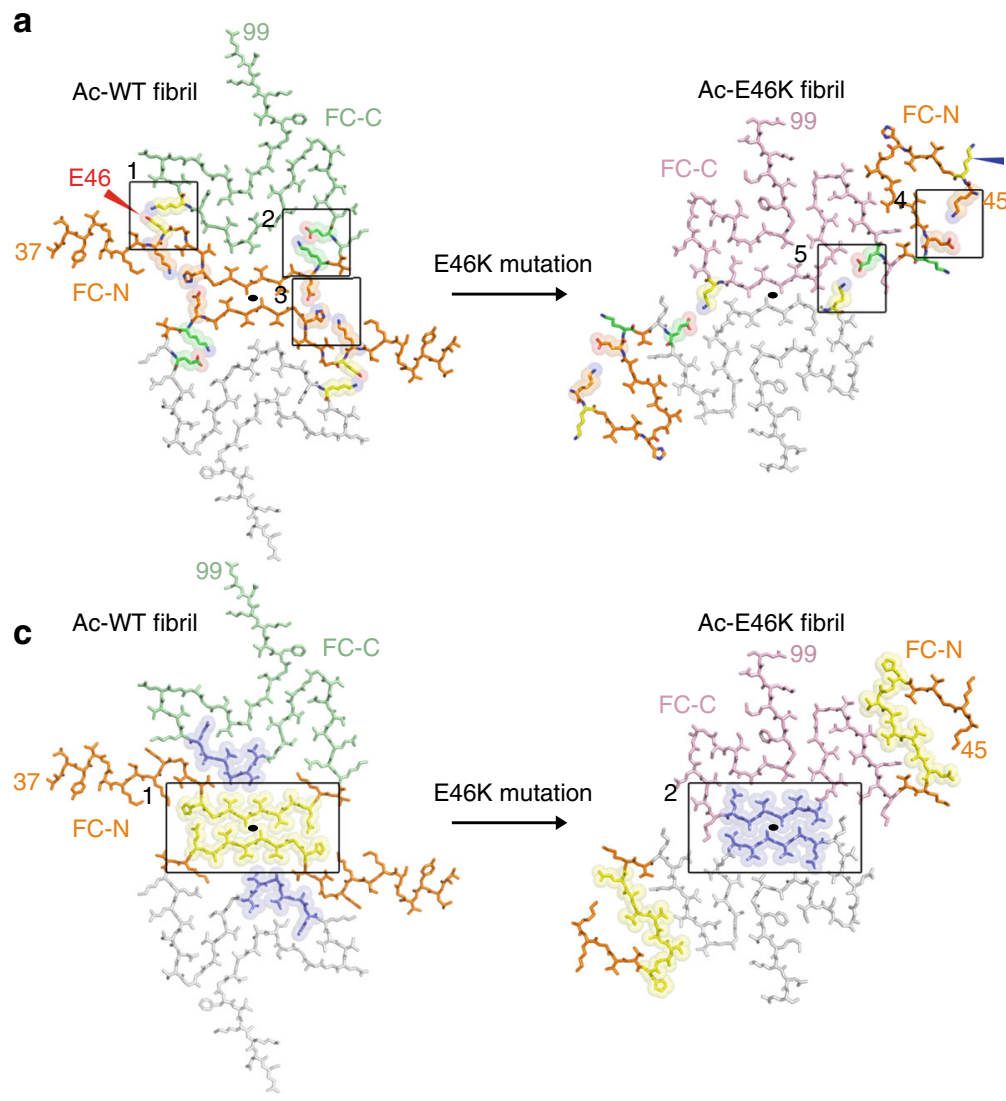

b

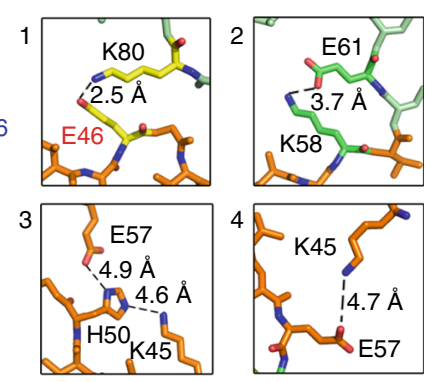

5

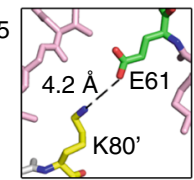

d
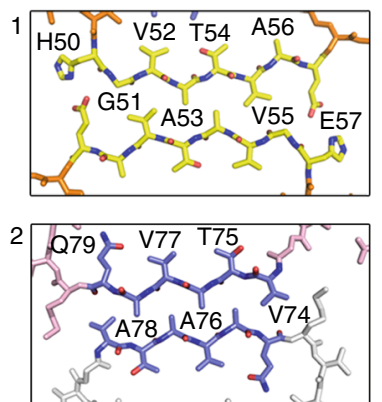

e

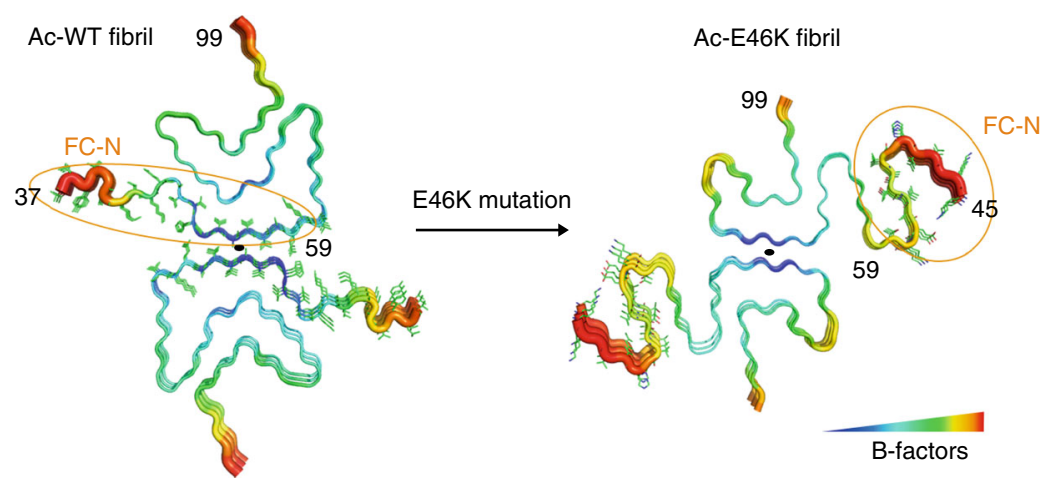

Fig. 4 Rearrangement of the $\boldsymbol{\alpha}$-syn fibril structure triggered by E46K mutation. a Electrostatic interactions in the Ac-WT and Ac-E46K fibrils. K/E pairs that form salt bridges are highlighted with spheres and shown in zoom-in views in (b). The FC-N region (residues 37/45-59) is colored in orange. The mutation site E46/K46 is highlighted in red/blue. The black solid ellipses in all panels indicate the symmetric axis of the dimeric $\alpha$-syn structure. $\mathbf{b}$ Zoom-in views of the corresponding regions in (a). Distances of the electrostatic interactions are indicated, and involved residues are labeled. The mutation site E46 is highlighted in red. c Protofilamental interfaces of the Ac-WT and Ac-E46K fibrils. Residues involved in the interfaces are highlighted with spheres. The fibril interface of Ac-WT fibril is colored in yellow; that of the Ac-E46K fibril is in blue. The interfaces are zoomed in (d). $\mathbf{d}$ Zoom-in views of the fibril interfaces. Interface residues are labeled. e Conformational change of the FC-N region results in a decreased stability of this region. Three layers of the $\alpha-$ syn fibrils are shown in B-factor putty. The FC-N region is shown in lines and highlighted with orange ellipses.

the Ac-E46K fibril reported in this work represents the only righthanded $\alpha$-syn fibril identified so far. Intriguingly, a recent study on $A \beta$ structure showed that brain-derived $A \beta$ fibrils are righthand twisted, rather than left-hand twisted as seen in the in vitro formed $A \beta$ fibrils ${ }^{35}$. What defines the helical chirality of the amyloid fibrils? Whether and how does it determine fibril pathology? These are interesting questions to be answered.

Protein structures are determined by their primary sequences. While, regarding amyloid proteins, this dogma becomes more complicated. The reported $\alpha$-syn fibril structures usually contain the central region of $\alpha$-syn including residues $\sim 37-99^{15,16,22-24,27}$. This $\sim 60$ a.a. long sequence can fold into various structures to construct polymorphic a-syn fibrils. Several WT $\alpha$-syn polymorphs have been observed in the in vitro prepared fibrils ${ }^{15,16,23}$ (Supplementary Fig. 9). Furthermore, a recent study on brainderived $\alpha$-syn fibril showed that in vivo cofactors can participate into a-syn fibril structure and introduce more polymorphs ${ }^{36}$. Recently, several fibril structures formed by inherited $\alpha$-syn mutants have bee reported including Ac-A53T ${ }^{37}, \mathrm{H}_{50 Q}{ }^{24}$, and E46K 23,38 . The Ac-A53T and H50Q fibrils exhibit similar morphology, and a-syn monomer in these structures folds similarly to that in the Ac-WT fibril (Supplementary Fig. 9). Two E46K a-syn fibril structures have been reported ${ }^{23,38}$. These two structures closely resemble the so-called polymorph 2a WT a-syn fibril 
$\alpha-\operatorname{Syn}(37-99)$

$\begin{array}{lllllll}43 \quad 4546 & 50 & 5758 & 6061 & 80 & 83 & 969798\end{array}$

VLYVGSKTKEGVVHGVATVAEKTKEQVTNVGGAVVTGVTAVAQKTVEGAGSIAAATGFVKKDQ

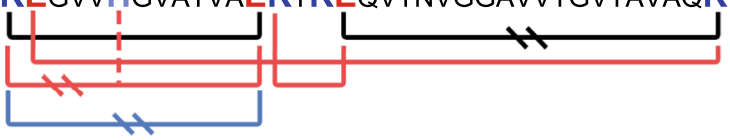

Ac-E46K fibril (6L4S)

Ac-WT fibril (6A6B)
WT fibril (6RT0)

$\rightarrow$ Inter-molecular interaction
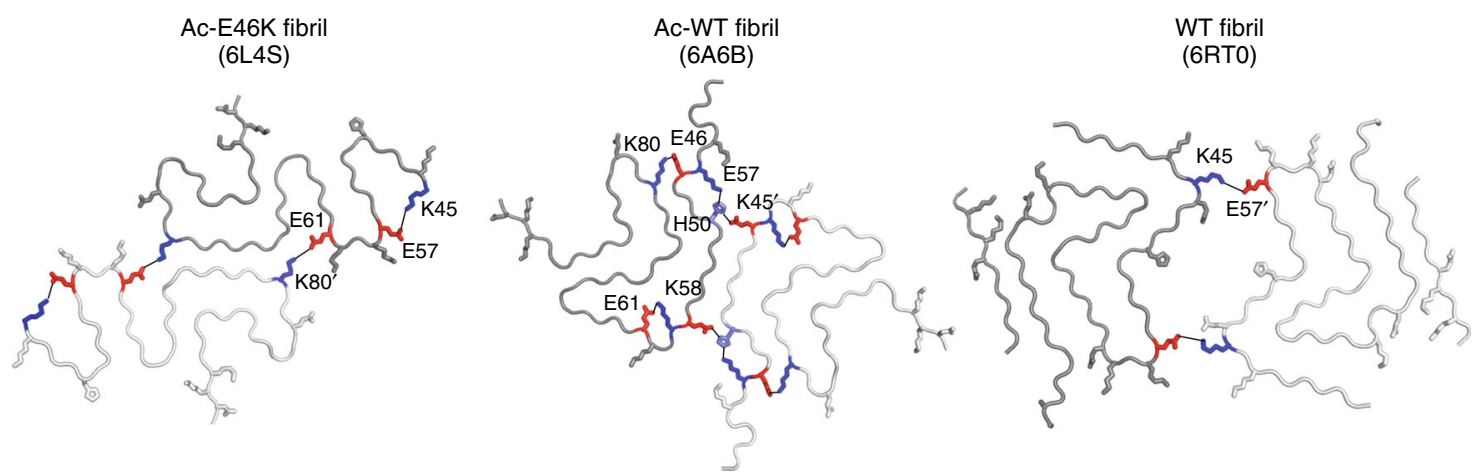

Fig. 5 Electrostatic interactions in $\boldsymbol{\alpha}$-syn fibril polymorphs. Primary sequence of WT $\alpha$-syn fibril core (FC) is shown on top. K residues are colored in blue; $\mathrm{H}$ is light blue; $\mathrm{E} / \mathrm{D}$ is red. Interacting charged residues in the structures of different $\alpha$-syn polymorphic fibrils are connected with solid lines. One layer of different polymorphic structures of $\alpha$-syn fibrils are shown below. The dimeric $\alpha$-syn molecules are colored in different levels of gray. Electrostatic interactions are highlighted with $\mathrm{K}$ colored in blue, $\mathrm{H}$ in light blue and $\mathrm{E}$ in red. PDB IDs of the structures are provided in parentheses.

(Supplementary Fig. 9). Distinctive from the known polymorphs, the Ac-E46K fibril structure in the current work represents a distinct fold of $\alpha$-syn (Supplementary Fig. 9), which demonstrates how the E46K mutation might rearrange the fibril structure. The polymorphic structures of $\mathrm{E} 46 \mathrm{~K}$ fibrils may derive from the different fibrillation conditions, and $\mathrm{N}$-terminal acetylation may also play a role by kinetically affecting aggregation ${ }^{39}$.

Then, how many fibril structures can $\alpha$-syn potentially form? What determines the possibilities? We notice that different $\alpha$-syn structures contain different electrostatic interactions, which play an important role in defining and stabilizing the overall structure (Fig. 5). In the Ac-WT fibril, intra-molecular electrostatic interactions of K58-E61 and E46-K80 lock the Greek key-like fold of a-syn, and the inter-molecular interactions of E45-H50-E57' are involved in stabilizing the fibril interface, which pairs $\alpha$-syn protofilaments to form mature fibrils (Fig. 5). In contrast, in the polymorph 2a WT fibril ${ }^{23}$, electrostatic interaction forms between $\mathrm{K} 45$ and $\mathrm{E} 57^{\prime}$, which mediates the dimerization of the protofilaments (Fig. 5). Alternatively, the Ac-E46K fibril contains an intramolecular electrostatic interaction between K45 and E57, which locks the $\beta$-hairpin fold of the FC-N region, and an intermolecular interaction between $\mathrm{E} 61$ and $\mathrm{K} 80^{\prime}$, which stabilizes the interface of the mutant fibril (Fig. 5). Different patterns of electrostatic interactions are also observed in other amyloid fibrils. For instance, tau fibrils derived from Pick's disease exhibit different electrostatic interactions from those from AZ and chronic traumatic encephalopathy (CTE) $)^{40-42}$ (Supplementary Fig. 10). These structural information suggest that different combinations of charged residues may serve as one of the key factors for the selection of fibril polymorph. In addition, mutations, posttranslational modifications and cofactors can further enrich the potential variations of $\alpha$-syn fibril structures.

\footnotetext{
Methods

Preparation of Ac-WT and Ac-E46K $\boldsymbol{\alpha}$-syn. Protein expression and purification of full-length $\alpha$-syn Ac-WT and Ac-E46K mutation follows the same protocol described previously ${ }^{15}$. Briefly, the WT and E46K $\alpha$-syn gene were inserted into pET22 vector and transformed into E. coli expression cell line BL21(DE3) (TransGen Biotech, Cat. \# CD601-02). Primer sequences used in this study are listed in Supplementary Table 1. Fission yeast N-acetyltransferase complex B was
}

co-expressed with $\alpha$-syn to obtain the $\mathrm{N}$-terminally acetylated $\alpha$-syn ${ }^{43} .1 \mathrm{mM}$ isopropyl-1-thio-D-galactopyranoside (IPTG) was used to induce protein expression at $37^{\circ} \mathrm{C}$ for $4 \mathrm{~h}$. Cells were harvested in $50 \mathrm{mM}$ Tris- $\mathrm{HCl}, \mathrm{pH} 8.0,1 \mathrm{mM}$ phenylmethylsulfonyl fluoride, $1 \mathrm{mM}$ EDTA and lysed by sonication. After centrifugation at $16000 \times \mathrm{g}$ for $30 \mathrm{~min}$, the supernatant was boiled at $100{ }^{\circ} \mathrm{C}$ for $10 \mathrm{~min}$. After another centrifugation, streptomycin $\left(20 \mathrm{mg} \mathrm{ml}^{-1}\right)$ was added to remove nucleic acid in the supernatant. $\mathrm{pH}$ was adjusted to 3.5 using $2 \mathrm{M} \mathrm{HCl}$ to precipitate other unwanted components. $50 \mathrm{mM}$ Tris- $\mathrm{HCl}(\mathrm{pH} 8.0)$ was used to dialyze the supernatants overnight. Q column (GE Healthcare, 17-5156-01) and sizeexclusion chromatography Superdex 75 (GE Healthcare, 28-9893-33) were used for protein purification. Protein samples were loaded to Q column with buffer A (50 mM Tris- $\mathrm{HCl}, \mathrm{pH} 8.0$ ), and eluted with a gradient (20-60\%) of buffer B (50 mM Tris- $\mathrm{HCl}, 1 \mathrm{M} \mathrm{NaCl}, \mathrm{pH} 8.0$ ). For Superdex75, the buffer containing $50 \mathrm{mM}$ Tris- $\mathrm{HCl}, 150 \mathrm{mM} \mathrm{KCl} \mathrm{pH8.0} \mathrm{was} \mathrm{used.} \mathrm{After} \mathrm{purification,} \mathrm{an} \mathrm{on-line}$ EASY-nL-LC 1000 coupled with an Orbitrap Q-Exactive HF mass spectrometer was used to verify that the Ac-E46K a-syn protein sample is indeed acetylation (Supplementary Fig. 1).

Fibrillation of Ac-WT and Ac-E46K $\boldsymbol{\alpha}$-syn. $100 \mu \mathrm{M}$ recombinant Ac-WT and AcE46K $\alpha$-syn in $50 \mathrm{mM}$ Tris, $\mathrm{pH} 7.5,150 \mathrm{mM} \mathrm{KCl}$ were incubated at $37^{\circ} \mathrm{C}$ with constant agitation $(900 \mathrm{rpm})$ in ThermoMixer (Eppendorf) for a week, respectively. The preformed fibrils were sonicated at $20 \%$ power for 15 times ( $1 \mathrm{~s}$ per time, $1 \mathrm{~s}$ interval) on ice by JY92-IIN sonicator (Xinyi Sonication Equipment Company, Ningbo, China). Then, the $\alpha$-syn PFF seeds $(0.5 \mathrm{~mol} \%)$ were added to $100 \mu \mathrm{M} \alpha$ syn monomer, and further incubated at $37^{\circ} \mathrm{C}$ with agitation (900 rpm) for a week to obtain mature fibrils.

CD spectroscopy. For cold incubation assay, $20 \mu \mathrm{M}$ Ac-WT or Ac-E46K a-syn fibrils were incubated at $0{ }^{\circ} \mathrm{C}$. Fibril samples were picked up at different incubation time for $\mathrm{CD}$ measurement. For freeze-thaw assay, fibril samples were incubated in liquid nitrogen for $10 \mathrm{~min}$ and then warmed up in water bath at room temperature for $5 \mathrm{~min}$. The secondary structure of the fibril sample was measured by a Chirascan CD spectrometer (Applied Photophysics, UK). Spectra were recorded from $200 \mathrm{~nm}$ to $260 \mathrm{~nm}$ at $0{ }^{\circ} \mathrm{C}$ or room temperature using a cell with a path length of $0.1 \mathrm{~cm}$. All spectra were collected more than 3 times with a background-corrected against buffer blank.

Atomic force microscopy (AFM). As for characterizing fibril fragmentation, $20 \mu \mathrm{M} \mathrm{Ac}$-WT or Ac-E46K $\alpha$-syn fibrils were sonicated for 4 times $(1 \mathrm{~s}$ per time, $1 \mathrm{~s}$ interval) on ice by JY92-IIN sonicator. $8 \mu \mathrm{l}$ of diluted samples $(10 \mu \mathrm{M})$ were mounted on a freshly cleaved mica for 3 min and gently rinsed with Milli-Q water to remove unbound fibrils. The samples were dried by nitrogen flow. Images were acquired with ScanAsyst air mode by using Nanoscope V Multimode 8 (Bruker). SNL-10 probe was taken with a constant of $0.35 \mathrm{~N} \mathrm{~m}^{-1}$ for scanning. Measurements were recorded at $512 \times 512$ pixels at a line rate at $1.5 \mathrm{~Hz}$. The percentage for different length of fibrils were processed and analyzed by using NanoScope Analysis software (version 1.5). 
As for characterizing the morphology of mature $\alpha$-syn fibrils, samples were acquired with $2 \mu \mathrm{M} \alpha$-syn fibrils. The image and height were analyzed on the Nanoscope software and imageJ.

Proteolytic digestion of $\alpha$-syn PFFs. $\alpha$-Syn fibrils were concentrated by centrifugation $\left(14,462 \times g, 25^{\circ} \mathrm{C}, 45 \mathrm{~min}\right)$, washed and resuspended with PBS, followed by sonication at $20 \%$ power for 15 times ( $1 \mathrm{~s}$ per time, $1 \mathrm{~s}$ interval) on ice by JY92IIN sonicator. $\alpha$-Syn PFFs $\left(3 \mathrm{mg} \mathrm{ml}^{-1}, 25 \mu \mathrm{l}\right.$, in PBS, PH 7.4) were incubated with proteinase $\mathrm{K}$ (final concentration $0.5,1,1.5,2,2.5 \mu \mathrm{g} \mathrm{ml}^{-1}$, Invitrogen) at $37^{\circ} \mathrm{C}$ for $30 \mathrm{~min}$. Reaction was terminated by adding $1 \mathrm{mM}$ PMSF. The samples were boiled with SDS-loading buffer for $15 \mathrm{~min}$ and loaded on $4-20 \%$ Bis-Tris gels (GenScript). The gels were stained by Coomassie brilliant blue and images were recorded and analyzed with Image Lab 3.0 (Bio-Rad)

ThT kinetic assay. $\alpha$-Syn PFF seeds were prepared by sonication at $20 \%$ power for 4 times ( $1 \mathrm{~s}$ per time, $1 \mathrm{~s}$ interval) on ice by JY92-IIN sonicator. The concentration of sonicated PFF seeds was calculated as equal to that of the PFFs before sonication. The concentration of PFFs before sonication was calculated as the amount of $\alpha$-syn monomer subtracting the amount of soluble $\alpha$-syn after fibril formation. $0.3 \mathrm{~mol} \%$ or $0.5 \mathrm{~mol} \% \alpha$-syn AcWT or Ac-E46K PFF seeds were added into $50 \mu \mathrm{M} \alpha$-syn monomer ( $50 \mathrm{mM}$ Tris, pH 7.5, $150 \mathrm{mM} \mathrm{KCl}, 0.05 \% \mathrm{NaN}_{3}$ ). Final concentration of $10 \mu \mathrm{M}$ ThT was added to the reaction mixture. Reactions were performed in triplicates in a 384 well optical plate (Thermo Scientific Nunc). The plate was detected by a Varioskan ${ }^{\circledast}$ Flash microplate reader (Thermo Scientific). Fluorescent intensities of each reaction were acquired at $440 \mathrm{~nm}$ (excitation) and $485 \mathrm{~nm}$ (emission) wavelengths, with a bottom read. Graphing was performed with GraphPad Prism 6 .

Negative-staining electron microscopy. Five microliters of aliquot of fibril sample was applied to a glow-discharged 200 mesh carbon support film (Beijing Zhongjingkeyi Technology Co., Ltd.) for $45 \mathrm{~s}$. Then the grid was washed with $5 \mu \mathrm{l}$ double-distilled water and followed by another wash of $5 \mu \mathrm{l} 3 \% \mathrm{w} / \mathrm{v}$ uranyl acetate. Another $5 \mu \mathrm{l} 3 \% \mathrm{w} / \mathrm{v}$ uranyl acetate was added to the grid to stain the sample for $45 \mathrm{~s}$. After removing the excess buffer by filter paper, the grid was dried by infrared lamp. The sample imaging was applied by Tecnai T12 microscope (FEI Company) operated at $120 \mathrm{KV}$.

Cryo-electron microscopy. An aliquot of $4 \mu \mathrm{l}$ of $6 \mu \mathrm{M}$ Ac-E46K $\alpha$-syn fibril solution was applied to a glow-discharged holey carbon grid (Quantifoil R1.2/1.3, $300 \mathrm{mesh}$ ), blotted for $6.5 \mathrm{~s}$, and plunge-frozen in liquid ethane using FEI Vitrobot Mark IV with $95 \%$ humidity at $16^{\circ} \mathrm{C}$. The grids were loaded onto a FEI Titan Krios microscope operated at $300 \mathrm{kV}$ with magnification of $\times 105,000$, in which a GIF Quantum energy filter (slit width $20 \mathrm{eV}$ ) were equipped. A Gatan K2 Summit camera recorded the cryo-EM micrographs using super-resolution mode with pixel size $0.665 \AA$. Defocus values were set from -2.4 to $-1.5 \mu \mathrm{m}$. All of the micrographs were dose-fractionated to 32 frames and the electron dose rate was set to $\sim 8.2$ counts per physical pixel per second $\left(\sim 6.25 \mathrm{e}^{-} \mathrm{s}^{-1}\right.$ per $\left.\AA^{2}\right)$, total exposure time is $8 \mathrm{~s}$, and $0.25 \mathrm{~s}$ per frame. Hence, the total dose is $\sim 50 \mathrm{e}^{-}$per $\AA^{2}$. AutoEMation2 written by Dr. Lei was used for all data collection ${ }^{44}$.

Image pre-processing. All 32 frames were aligned, summed and dose-weighted by MotionCorr2 and further binned to a pixel size of $1.33 \AA^{45}$. The defocus values of dose-weighted micrographs were estimated by CTFFIND4.1.5 ${ }^{46}$, and micrographs with the highest-resolution Thon ring $>6 \AA$, were kept for the subsequent processing in RELION3.0 ${ }^{47}$. 13,064 filaments were picked manually from 754 micrographs. The extracted segments (box size: $160 \times 160$, inter-box distance of $19 \AA$ A ) were classified by 2D (two-dimensional) classification. Segments of classes that have clear cross- $\beta$ strands were kept, and a steric-zipper conformation exists which is the same with the conformation of the wild type of $\alpha$-syn fibril.

Helical parameters determination. Twist angle and rise value of helical polymer was calculated before reconstruction. Micrographs, which were involved in the selected $2 \mathrm{D}$ classes, were used to estimate the rise value by a $\mathrm{C}++$ program that searches the strongest diffraction position around the $1 / 4.8 \AA^{-1}$ in Fourier space. The average of these positions was converted to the rise value in real space. The way to estimate the twist angle was the same as that described in our previous structural determination of Ac-WT a-syn fibril ${ }^{15}$, in which helical pitch length of each filament was estimated and the average of these helical pitch lengths was used to calculate the twist angle.

Helical reconstruction. Several iterations of 2D classification, 3D classification and $3 \mathrm{D}$ refinement were performed to filter the segments belonging to the same conformation. Segments of the selected 2D classes were used to reconstruct a reference with more feature in 3D refinement from the initial featureless cylinder model created by the relion_helix_toolbox program. 3D classification $(\mathrm{k}=2)$ then built a reasonable map based on the improved reference model. Auto-refine procedure was used using the single class with higher resolution from the result of $3 \mathrm{D}$ classification. No explicit -tau2_fudge value was specified, and no mask was used. The final map was convergence with the rise of $2.38 \AA$ and the twist angle of $-179.37^{\circ}$. The map was sharpened with a B-factor of $-97.8051 \AA^{2}$ using postprocessing subroutine in RELION. The overall resolution was reported at $3.37 \AA$ by the gold-standard FSC $=0.143$ criteria. Local resolution estimation calculated by ResMap ${ }^{48}$.

Polymorphs of Ac-E46K $\boldsymbol{\alpha}$-syn fibril. Two polymorphs at least existed in micrographs. One is twisted filament, and the another one is non-twisted filament which currently cannot be solved since this polymorph has no twist. All filament including twisted and non-twisted filaments in 754 micrographs were count as 18891. Among them, 13064 was twisted filaments which was used for reconstruction. The percentage of twisted filament is $69.15 \%$ and was dominated in the two fibril polymorphs.

Model building and refinement. A homology model based on the NMR structure (PDB entry code $2 \mathrm{~N} 0 \mathrm{~A}$ ) was built and modified by $\mathrm{COOT}^{28,49}$. The model with three adjacent layers (six promoters) was refined using the real-space refinement program in PHENIX ${ }^{50}$. The subunit dimers in the middle of three layers was extracted and used as the final model.

Reporting summary. Further information on research design is available in the Nature Research Reporting Summary linked to this article.

\section{Data availability}

Density maps of the Ac-E46K $\alpha$-syn fibril are available through EMDB with entry code: EMD-0833. The structural model was deposited in the Protein Data Bank with entry code: 6L4S. Other structural models used in this study are available in the Protein Data Bank with entry codes: 6A6B (Ac-WT), 6RT0 (WT polymorph 2a), 6RTB (WT polymorph 2b), 6CU8 (WT), 6LRQ (Ac-A53T), 6PES (H50Q), 6UFR (E46K), and 2N0A (solid-state NMR structure of WT). The source data underlying Figs. 1a, 1b, 1e, If and Supplementary Fig. $5 \mathrm{~d}$ are provided as a Source Data file. Other data that support the findings of this study are available from the corresponding authors upon reasonable request.

Received: 12 November 2019; Accepted: 28 April 2020; Published online: 26 May 2020

\section{References}

1. Woerman, A. L. et al. alpha-synuclein: multiple system atrophy prions. Cold Spring Harb. Perspect. Med. 8, a024588 (2018).

2. Spillantini, M. G. \& Goedert, M. The alpha-synucleinopathies: Parkinson's disease, dementia with Lewy bodies, and multiple system atrophy. Ann. N. Y Acad. Sci. 920, 16-27 (2000).

3. Spillantini, M. G. et al. Alpha-synuclein in Lewy bodies. Nature 388, 839-840 (1997).

4. Kim, S. et al. Transneuronal propagation of pathologic alpha-synuclein from the gut to the brain models Parkinson's disease. Neuron 103, 627-641 e7 (2019)

5. Luk, K. C. et al. Pathological alpha-synuclein transmission initiates Parkinson-like neurodegeneration in nontransgenic mice. Science $\mathbf{3 3 8}$ 949-953 (2012).

6. Mao, X. et al. Pathological alpha-synuclein transmission initiated by binding lymphocyte-activation gene 3. Science 353, aah3374 (2016).

7. Prusiner, S. B. et al. Evidence for alpha-synuclein prions causing multiple system atrophy in humans with parkinsonism. Proc. Natl Acad. Sci. USA 112, E5308-E5317 (2015).

8. Chartier-Harlin, M. C. et al. Alpha-synuclein locus duplication as a cause of familial Parkinson's disease. Lancet 364, 1167-1169 (2004).

9. Singleton, A. B. et al. alpha-synuclein locus triplication causes Parkinson's disease. Science 302, 841 (2003).

10. Lesage, S. et al. G51D alpha-synuclein mutation causes a novel parkinsonianpyramidal syndrome. Ann. Neurol. 73, 459-471 (2013).

11. Zarranz, J. J. et al. The new mutation, E46K, of alpha-synuclein causes Parkinson and Lewy body dementia. Ann. Neurol. 55, 164-173 (2004).

12. Polymeropoulos, M. H. et al. Mutation in the alpha-synuclein gene identified in families with Parkinson's disease. Science 276, 2045-2047 (1997).

13. Inigo-Marco, I. et al. E46K alpha-synuclein pathological mutation causes cellautonomous toxicity without altering protein turnover or aggregation. Proc. Natl Acad. Sci. USA 114, E8274-E8283 (2017).

14. Lee, M. K. et al. Human alpha-synuclein-harboring familial Parkinson's disease-linked Ala-53-> Thr mutation causes neurodegenerative disease with alpha-synuclein aggregation in transgenic mice. Proc. Natl Acad. Sci. USA 99, 8968-8973 (2002).

15. Li, Y. et al. Amyloid fibril structure of alpha-synuclein determined by cryoelectron microscopy. Cell Res. 28, 897-903 (2018). 
16. Li, B. et al. Cryo-EM of full-length alpha-synuclein reveals fibril polymorphs with a common structural kernel. Nat. Commun. 9, 3609 (2018).

17. Prots, I. et al. alpha-Synuclein oligomers induce early axonal dysfunction in human iPSC-based models of synucleinopathies. Proc. Natl Acad. Sci. USA 115, 7813-7818 (2018)

18. Greenbaum, E. A. et al. The E46K mutation in alpha-synuclein increases amyloid fibril formation. J. Biol. Chem. 280, 7800-7807 (2005).

19. Lemkau, L. R. et al. Site-specific perturbations of alpha-synuclein fibril structure by the Parkinson's disease associated mutations A53T and E46K. PLoS ONE 8, e49750 (2013).

20. de Oliveira, R. M. et al. The mechanism of sirtuin 2-mediated exacerbation of alpha-synuclein toxicity in models of Parkinson disease. PLoS Biol. 15, e2000374 (2017).

21. Theillet, F. X. et al. Structural disorder of monomeric alpha-synuclein persists in mammalian cells. Nature 530, 45-50 (2016).

22. Guerrero-Ferreira, R. et al. Cryo-EM structure of alpha-synuclein fibrils. eLife 7, e36402 (2018)

23. Guerrero-Ferreira, R. et al. Two new polymorphic structures of human fulllength alpha-synuclein fibrils solved by cryo-electron microscopy. eLife $\mathbf{8}$ e48907 (2019).

24. Boyer, D. R. et al. Structures of fibrils formed by alpha-synuclein hereditary disease mutant $\mathrm{H} 50 \mathrm{Q}$ reveal new polymorphs. Nat. Struct. Mol. Biol. 26, 1044-1052 (2019).

25. Ikenoue, T. et al. Cold denaturation of alpha-synuclein amyloid fibrils. Angew. Chem. Int Ed. Engl. 53, 7799-7804 (2014).

26. Xue, W. F., Homans, S. W. \& Radford, S. E. Systematic analysis of nucleationdependent polymerization reveals new insights into the mechanism of amyloid self-assembly. Proc. Natl Acad. Sci. USA 105, 8926-8931 (2008).

27. Ni, X., McGlinchey, R. P., Jiang, J. \& Lee, J. C. Structural insights into alphasynuclein fibril polymorphism: effects of Parkinson's disease-related cterminal truncations. J. Mol. Biol. 431, 3913-3919 (2019).

28. Tuttle, M. D. et al. Solid-state NMR structure of a pathogenic fibril of fulllength human alpha-synuclein. Nat. Struct. Mol. Biol. 23, 409-415 (2016)

29. Fitzpatrick, A. W. \& Saibil, H. R. Cryo-EM of amyloid fibrils and cellular aggregates. Curr. Opin. Struct. Biol. 58, 34-42 (2019).

30. Qiang, W., Yau, W. M., Lu, J. X., Collinge, J. \& Tycko, R. Structural variation in amyloid-beta fibrils from Alzheimer's disease clinical subtypes. Nature 541, 217-221 (2017).

31. Liu, C. et al. Out-of-register beta-sheets suggest a pathway to toxic amyloid aggregates. Proc. Natl Acad. Sci. USA 109, 20913-20918 (2012).

32. Peng, C. et al. Cellular milieu imparts distinct pathological alpha-synuclein strains in alpha-synucleinopathies. Nature 557, 558-563 (2018).

33. Goedert, M., Eisenberg, D. S. \& Crowther, R. A. Propagation of tau aggregates and neurodegeneration. Annu Rev. Neurosci. 40, 189-210 (2017).

34. Peelaerts, W. et al. alpha-synuclein strains cause distinct synucleinopathies after local and systemic administration. Nature 522, 340-344 (2015).

35. Kollmer, M. et al. Cryo-EM structure and polymorphism of $A \beta$ amyloid fibrils purified from Alzheimer's brain tissue. Nat. Commun. 10, 4760 (2019).

36. Schweighauser, M. et al. Structures of $\alpha$-synuclein filaments from multiple system atrophy. Preprint at https://www.biorxiv.org/content/10.1101/ 2020.02.05.935619v1 (2020).

37. Sun, Y. et al. Cryo-EM structure of full-length $\alpha$-synuclein amyloid fibril with Parkinson's disease familial A53T mutation. Cell Res. 30, 360-362 (2020).

38. Boyer, D. R. et al. The $\alpha$-synuclein hereditary mutation E46K unlocks a more stable, pathogenic fibril structure. Proc. Natl Acad. Sci. USA 117, 3592-3602 (2020).

39. Watson, M. D. \& Lee, J. C. N-terminal acetylation affects $\alpha$-synuclein fibril polymorphism. Biochemistry 58, 3630-3633 (2019).

40. Falcon, B. et al. Novel tau filament fold in chronic traumatic encephalopathy encloses hydrophobic molecules. Nature 568, 420-423 (2019).

41. Falcon, B. et al. Structures of filaments from Pick's disease reveal a novel tau protein fold. Nature 561, 137-140 (2018).

42. Fitzpatrick, A. W. P. et al. Cryo-EM structures of tau filaments from Alzheimer's disease. Nature 547, 185-190 (2017).

43. Johnson, M., Coulton, A. T., Geeves, M. A. \& Mulvihill, D. P. Targeted aminoterminal acetylation of recombinant proteins in E. coli. PLoS ONE 5, e15801 (2010).

44. Lei, J. \& Frank, J. Automated acquisition of cryo-electron micrographs for single particle reconstruction on an FEI Tecnai electron microscope. J. Struct. Biol. 150, 69-80 (2005).
45. Zheng, S. Q. et al. MotionCor2: anisotropic correction of beam-induced motion for improved cryo-electron microscopy. Nat. Methods 14, 331-332 (2017).

46. Rohou, A. \& Grigorieff, N. CTFFIND4: Fast and accurate defocus estimation from electron micrographs. J. Struct. Biol. 192, 216-221 (2015).

47. Zivanov, J. et al. New tools for automated high-resolution cryo-EM structure determination in RELION-3. elife 7, e42166 (2018)

48. Kucukelbir, A., Sigworth, F. J. \& Tagare, H. D. Quantifying the local resolution of cryo-EM density maps. Nat. Methods 11, 63-65 (2014).

49. Emsley, P. \& Cowtan, K. Coot: model-building tools for molecular graphics. Acta Crystallogr D: Biol. Crystallogr 60, 2126-2132 (2004).

50. Adams, P. D. et al. PHENIX: a comprehensive Python-based system for macromolecular structure solution. Acta Crystallogr D: Biol. Crystallogr 66 , 213-221 (2010).

\section{Acknowledgements}

This work was supported by the Major State Basic Research Development Program (2016YFA0501902), the Science and Technology Commission of Shanghai Municipality (18JC1420500), "Eastern Scholar" project supported by Shanghai Municipal Education Commission, the Shanghai Pujiang Program (18PJ1404300), the National Natural Science Foundation (NSF) of China (91853113, 31872716, and 31800709), the Shanghai Municipal Science and Technology Major Project (2019SHZDZX02), and the Advanced Innovation Center for Structural Biology, Tsinghua-Peking Joint Center for Life Sciences. We acknowledge Tsinghua University Branch of China National Center for Protein Sciences Beijing and the cryo-EM platform of Peking University for providing facility supports in cryo-EM.

\section{Author contributions}

C.L., X.L., and D.L. designed the project. K.Z., H.L., C.Z., and F.L. prepared the Ac-WT and Ac-E46K monomer and fibril samples. Z.L., H.L., K.Z., and Y.T. performed the biochemical assays. Z.L. and Y.S. performed the AFM experiments. Y.L, K.Z. and X-D.S performed the cryo-EM experiments. Y.L. built and refined the structure model. All of the authors are involved in analyzing the data and contributed to manuscript discussion and editing. D.L. and C.L. wrote the manuscript.

\section{Competing interests}

The authors declare no competing interests.

\section{Additional information}

Supplementary information is available for this paper at https://doi.org/10.1038/s41467 020-16386-3.

Correspondence and requests for materials should be addressed to D.L., X.L. or C.L.

Peer review information Nature Communications thanks the anonymous reviewer(s) for their contribution to the peer review of this work.

Reprints and permission information is available at http://www.nature.com/reprints

Publisher's note Springer Nature remains neutral with regard to jurisdictional claims in published maps and institutional affiliations.

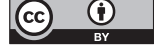

Open Access This article is licensed under a Creative Commons Attribution 4.0 International License, which permits use, sharing, adaptation, distribution and reproduction in any medium or format, as long as you give appropriate credit to the original author(s) and the source, provide a link to the Creative Commons license, and indicate if changes were made. The images or other third party material in this article are included in the article's Creative Commons license, unless indicated otherwise in a credit line to the material. If material is not included in the article's Creative Commons license and your intended use is not permitted by statutory regulation or exceeds the permitted use, you will need to obtain permission directly from the copyright holder. To view a copy of this license, visit http://creativecommons.org/ licenses/by/4.0/.

(C) The Author(s) 2020 